Relations industrielles

Industrial Relations

\title{
L'infirmière et son perfectionnement. Rédigé en collaboration. Cahiers Leannec, no 4, 1960. P. Lethilleux, 10, rue Casette, Paris. 72 pp.
}

\section{L.}

Volume 16, numéro 2, avril 1961

URI : https://id.erudit.org/iderudit/1021821ar

DOI : https://doi.org/10.7202/1021821ar

Aller au sommaire du numéro

Éditeur(s)

Département des relations industrielles de l’Université Laval

ISSN

0034-379X (imprimé)

1703-8138 (numérique)

Découvrir la revue

Citer ce compte rendu

L., M. (1961). Compte rendu de [L'infirmière et son perfectionnement. Rédigé en collaboration. Cahiers Leannec, no 4, 1960. P. Lethilleux, 10, rue Casette, Paris. 72 pp.] Relations industrielles / Industrial Relations, 16(2), 275-276.

https://doi.org/10.7202/1021821ar

Tous droits réservés (C Département des relations industrielles de l’Université Laval, 1961
Ce document est protégé par la loi sur le droit d'auteur. L'utilisation des services d'Érudit (y compris la reproduction) est assujettie à sa politique d'utilisation que vous pouvez consulter en ligne.

https://apropos.erudit.org/fr/usagers/politique-dutilisation/ 
Urbana, 1958, 170 pp., $\$ 3.50$.

L'analyse du marché du travail est conçue différemment par deux écoles. La première qu'on pourrait qualifier d'école économique pure postule que les travailleurs changent d'occupations à partir d'une considération rationnelle des différences dans les taux de salaire. Dans cette optique, on s'attache uniquement à l'analyse du salaire et on néglige les effets des institutions sociales sur les variations du marché du travail. La deuxième école soutient que la théorie économique classique ne peut rendre compte adéquatement de la mobilité des travailleurs. Pour les tenants de cette école l'analyse de la structure des salaires est secondaire dans l'explication du marché du travail. Les facteurs principaux d'explication sont les institutions sociales (en particulier le syndicalisme) et la motivation irrationnelle des travailleurs. Certains auteurs vont même jusqu'à établir une distinction complète entre le \&marché des salaires》 et le «marché des emplois 》.

La position des auteurs de ces monographies est intermédiaire. Ils acceptent le fait que la mobilité des travailleurs est causée en partie par des variables non-économiques mais prétendent que les facteurs économiques (salaires) ont quand même une importance qu'on ne peut négliger dans une étude scientifique.

A partir de cette prise de position, les auteurs élaborent une série d'hypotheses concernant le comportement des travailleurs et des employeurs. Ce sont ces hypothèses qu'ils ont voulu vérifier par l'étude monographique de deux marchés du travail situés en dehors des zones métropolitaines. Leur conclusion générale est que l'analyse économique ne peut plus prétendre rendre compte à elle seule des fluctuations du marché du travail. Cette analyse doit être complétée par des analyses sociologiques et psychologiques. On ne pourra rendre compte de la mobilité des travailleurs que si économistes, sociologues et psychologues combinent leurs optiques dans la recherche.

L'ouvrage de Wilcock et Sobel est très bien construit au point de vue méthodologique et scientifique. D'une position claire du problème, ils savent élaborer des hypothèses précises. Le travail de recherche empirique a été conduit de façon rigoureuse. Dans un style concis et très précis, ils rapportent l'essentiel de leurs données et des résultats.

Parce que le texte est très dense, sa lecture est un peu difficile, mais cette ouvrage mérite d'être lu attentivement à cause de sa haute valeur scientifique.

\section{Gérald Fortin.}

L'infirmière et son perfectionnement. Rédigé en collaboration. Cahiers Leannec, no 4,1960 . P. Lethilleux, 10 , rue Casette, Paris. 72 pp.

Dans tous les pays, on souffre d'une pénurie d'infirmières. Cela est dû pour une part à la progression rapide des services médicaux et, d'autre part, à l'abandon de la profession par les infirmières qualifiées. Ainsi, au Canada, on estimait que sur le total des infirmières diplômées agréées au 31 décembre 1955, $38.4 \%$ avaient renonçé à leur activité ou travaillaient dans d'autres domaines. Comment cela s'explique-t-il?

C'est pour donner une réponse à ces questions que les Cahiers Laënnec viennent de faire paraître un numéro consacré essentiellement à l'Infirmière et son perfectionnement.

L'article principal sur cla condition de l'infirmière » explique comment la croissance rapide de la profession pose avec acuité le problème non seulement des salaires, mais aussi du recrutement, de l'horaire de travail, de la promotion et de la spécialisation. Dans les articles suivants, les différents auteurs envisagent le perfectionnement de l'infirmière hospitalière ou soignante à domicile, et de la monitrice d'école. Plusieurs moyens pour promouvoir un enseignement post-scolaire sont décrits en dé tail: session intensive, cours magistraux, présentation de films.

En conclusion, une étude sur la profession de kinésithérapeute donne un aperçu très complet d'une nouvelle profession para-médicale appelée à un avenir certain.

Ce Cahier intéressera non seulement les infirmières, mais aussi les administrateurs d'hôpitaux et cliniques. A l'heure 
où le développement sanitaire du pays se trouve dangereusement freiné par le manque croissant de personnel qualifié pour les soins, une telle étude ne se contente pas d'être un cri d'alarme, elle propose aussi des éléments de solution.

\section{L.}

The Israeli Worker, by Ferdynand Zweig, Herzl Press and Sharon Books, New York, 1959, 305 pp., \$5.00.

L'auteur, avant de visiter Israël, croyait $\mathbf{y}$ trouver les mêmes genres de relations de travail et d'attitudes que dans les autres pays de l'Occident. Au contraire, nous dit-il, en Israël, tout est neuf, étrange, unique. Autant le paysage est riche et varié, autant le monde ouvrier est disparate et mouvant. Chaque groupe de travailleurs a ses problèmes particuliers de même que sa façon originale d'y apporter des solutions. Il est d'ailleurs d'autant plus difficile d'étudier ce monde du travail, qu'il est à peine naissant. Il n'existe aucune tradition dans ce domaine. Tout est à faire et des solutions nouvelles sont inventées à chaque jour.

L'état d'Israël doit trouver des moyens rapides pour assurer la prospérité du pays. L'industrialisation semble une des voies les plus sûres, mais elle suppose une expérience que ne possède pas la population. Une faible minorité des immigrants juifs sont des travailleurs manuels. Les ouvriers israéliens sont d'anciens collets blancs pour la plupart. Non seulement doivent-ils apprendre de nouvelles techniques, mais ils doivent aussi apprendre à devenir une nouvelle classe sociale. Ils doivent apprendre à avoir d'eux-mêmes une nouvelle image et à accepter cette nouvelle image.

Dans une société où s'affrontent le traditionalisme judaïque et un socialisme radical, une population autochtone et un flot constant d'immigrants, où le chômage est élevé et la sécurité d'em- ploi longue à obtenir, des conflits sont inévitables. Ces conflits, on cherche à les éviter par plusieurs structures plus ou moins conciliables. Socialisme, coopératisme, syndicalisme, co-gestion, entreprise privée, coexistent et forment un tout plus ou moins harmonieux. L'unité, du point de vue des travailleurs du moins, est assurée par le Histadrut, la Fédération générale des travailleurs juifs. Cette Fédération qui groupe à la fois des coopératives, des syndicats et des partis politiques, est pratiquement un état dans l'état. Elle contrôle la législation du travail, à la fois sur le plan législatif et sur le plan exécutif. De plus elle possède elle-même un bon nombre d'industries, réalisant ainsi le rêve socialiste de l'usine propriété des ouvriers.

C'est cette image complexe que monsieur Zweig essaie de nous présenter dans son ouvrage. Il réussit très bien à nous faire sentir la complexité de la situation. Mais une fois la lecture terminée, on se demande si la composition du volume ne rend pas la situation plus complexe qu'elle ne l'est en réalité. En effet on passe constamment de notions micro-sociologiques propre à la sociologie industrielle à des vues globales sur la société. Ni l'une ni l'autre de ces deux démarches n'étant assez systématiques pour donner une vue exacte des problèmes. Par ailleurs, il suppose trop de choses connues chez le lecteur. Ainsi le Histadrut est mentionné dès les premières pages mais il faut attendre à la page 238 pour en avoir une définition précise.

Malgré ces réserves sur la forme, l'ouvrage demeure très utile. Non seulement on peut en tirer des informations pertinentes sur la structure des occupations et sur les problèmes propres à certains types de travailleurs mais l'analyse du mouvement ouvrier israélien peut suggérer des réformes qui seraient utiles dans notre milieu.

GérALd Fortin. 